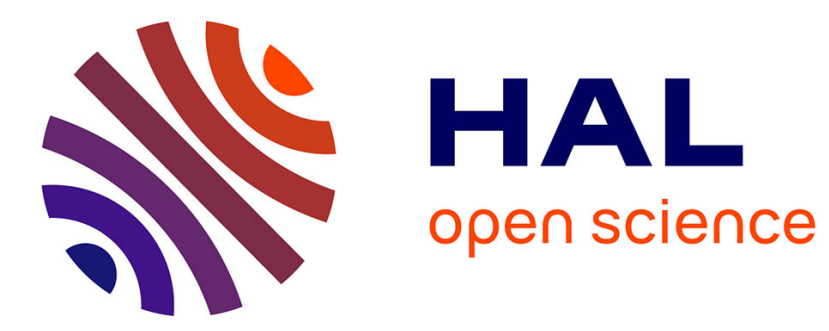

\title{
Énergie moyenne d'ionisation dans les gaz. Défaut d'ionisation
}

\author{
Robert Génin
}

\section{To cite this version:}

Robert Génin. Énergie moyenne d'ionisation dans les gaz. Défaut d'ionisation. J. Phys. Radium, 1956, 17 (7), pp.571-572. 10.1051/jphysrad:01956001707057100 . jpa-00235476

\section{HAL Id: jpa-00235476 https://hal.science/jpa-00235476}

Submitted on 1 Jan 1956

HAL is a multi-disciplinary open access archive for the deposit and dissemination of scientific research documents, whether they are published or not. The documents may come from teaching and research institutions in France or abroad, or from public or private research centers.
L'archive ouverte pluridisciplinaire HAL, est destinée au dépôt et à la diffusion de documents scientifiques de niveau recherche, publiés ou non, émanant des établissements d'enseignement et de recherche français ou étrangers, des laboratoires publics ou privés. 


\title{
ENERGIE MOYENNE D'IONISATION DANS LES GAZ. DÉFAUT D'IONISATION
}

\author{
Par Robert GENIN, \\ Laboratoire des Rayons X, École Pratique des Hautes Etudes, Paris.
}

\begin{abstract}
Sommaire. - L'énergie moyenne d'ionisation, pour les particules 'alpha du Plutonium, est déterminée dans l'air, l'azote, l'argon et le néon. Le défaut d'ionisation, analogue à la quantité définie par Knipp et Ling dans leur étude sur l'ionisation produite par les particules chargées lourdes, et qui peut être utilisé pour caractériser les écarts à la proportionnalité entre l'énergie et l'ionisation, est donné pour les particules alpha dans l'air.
\end{abstract}

Abstract. - The average energy required to produce an ion pair is determined, for $\mathrm{Pu} \alpha$ particles, in air, $\mathrm{N}_{2}, \mathrm{~A}$ and Ne. The ionization defect, analogous to the quantity defined by Knipp et Ling in their calculation on the ionization produced by heavy charged particles, is given for $\alpha$ particles in air.

I. La proportionnalité, au moins approximative, qui existe entre l'énergie $E$ d'une particule chargée et le nombre d'ions $I$ qu'elle produit en perdant cette énergie dans un gaz amène à définir la quantité $W=\frac{E}{I}$ comme l'énergie moyenne d'ionisation.

Pour la détermination de $\mathrm{W}$ dans différents gaz, j'ai utilisé, pour produire l'ionisation, les particules alpha du Plutonium $(E=5,140 \mathrm{MeV})$. Les ions produits dans une chambre d'ionisation à géométrie plane sont collectés, l'impulsion de tension qui en résulte est amplifiée et sa hauteur, à la sortie de l'amplificateur, est déterminée par un sélecteur d'amplitude à 100 canaux. Pour éviter les erreurs dues à la recombinaison en colonne, j'ai employé la méthode utilisée par F. Alder, P. Huber, F. Metzger (Helo. Phys. Acta, 1947, 20, 234,) qui consiste à effectuer la mesure de $\mathrm{W}$ pour diverses valeurs de l'intensité du champ électrique collectant les ions. D'après la théorie, due à Jaffé, de la recombinaison en colonne, l'inverse de l'ionisation, quantité proportionnelle à $W$, est une fonction linéaire de l'inverse du champ électrique $X$. La relation $W=f\left(\frac{1}{X}\right)$ doit donc être une droite, c'est la valeur extrapolée pour $\frac{1}{X}=0$, c'est-à-dire pour un champ infini qu'on prend pour énergie moyenne d'ionisation.

2. Résultats. - J'ai étudié les quatre gaz : air, azote, argon et néon - et obtenu les résultats suivants :

$\begin{array}{lccc} & \alpha \text { du Pu, } W \text { en eV } & \alpha \text { du Po (a) } & \alpha \text { du Po (b) } \\ \text { Air } & 35,5 \pm 0,5 & 35,5 & 34,7 \pm 0,5 \\ \text { Azote } & 36,6 \pm 0,5 & 36,6 & 36,3 \pm 0,4 \\ \text { Argon } & 26,5 \pm 0,3 & 26,4 & \\ \text { Néon } & 33,1 & 36,8 & \end{array}$

(a) Jesse (W. P.), Sadauskis (J.), Phys. Rev., 1953, 90, 1120.

(b) Alder (F.), Huber (P.), Metzger (F.), Helv. Phys. Acta, 1947, 20, 234.
Le désaccord pour le néon peut s'interpréter par la présence d'impuretés susceptibles de fournir des ions lorsqu'un atome de $\mathrm{Ne}$ excité revient à l'état fondamental en subissant un choc de seconde espèce contre une molécule de l'impureté. La valeur

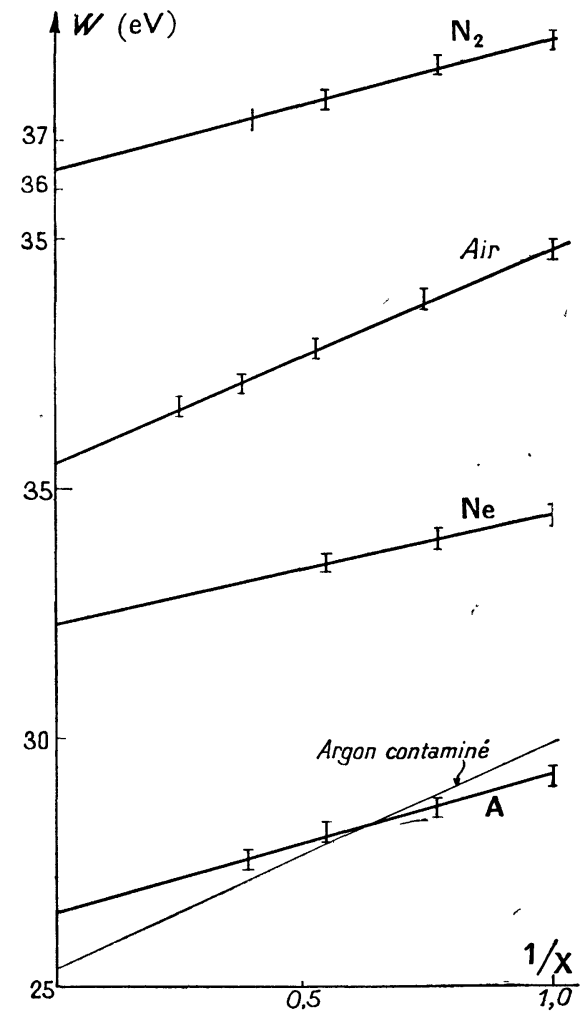

Fig. 1.

que j'ai obtenue est d'ailleurs intermédiaire entre le résultat de Jesse et Stadauskis et le résultat plus ancien de Stetter (Z. Physik., 1943, 120, 639) : $W_{\text {ve }}=29,8 \mathrm{eV}$.

Un phénomène analogue se produit lorsqu'on laisse l'argon séjourner longtemps dans la chambre. La droite de Jaffé s'incline davantage et la valeur 
de l'énergie moyenne d'ionisation s'abaisse de $4 \%$ environ.

3) Lorsqu'on détermine l'ionisation I produite par divers émetteurs $\alpha$, on peut tracer une partie de la courbe ionisation énergie qui, pour l'air et l'argon, est une droite du $\mathrm{Ra}(4,79 \mathrm{MeV})$ au $\mathrm{Th} C^{\prime}$

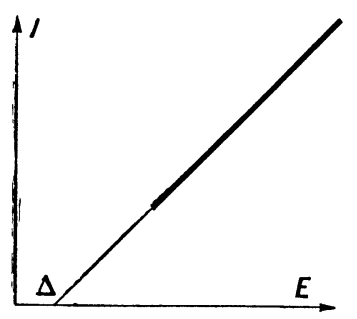

Fig. 2.

$(8,78 \mathrm{MeV})$. Si l'ionisation est proportionnelle à l'énergie, cette droite doit passer par l'origine; au contraire, s'il existe un écart à la loi de proportionnalité, la droite coupe l'axe des énergies en un point d'abscisse $\Delta$. L'énergie $\Delta$ peut done servir à caractériser de façon semi-quantitive les écarts à la proportionnalité énergie-ionisation qui existent dans un gaz donné. Par analogie, avec une quantité analogue introduite par J. K. Knipp et R. G Ling (Phys. Res., 1951, 82, 30) dans leur étude de l'ionisation des fragments de fission, on peut appeler $\Delta$ le défaut d'ionisation.
L'extrapolation de la droite expérimentale est trop aléatoire pour déterminer $\Delta$. Une méthode un peu plus précise consiste à déterminer $I / E$ pour diverses valeurs de $E$. En opérant ainsi, je n'ai trouvé aucune variation significative du rapport dans l'argon, alors que dans l'air, il subit un accroissement de $2 \%$ environ quand on passe du $\mathrm{Ra}$ au $\mathrm{Th} C^{\prime}$, ce qui conduit pour $\Delta$ à $200 \mathrm{keV}$ environ.

Il est peut-être intéressant de remarquer qu'on peut évaluer $\Delta$ autrement. Si la relation énergieionisation reste linéaire aux grandes énergies, $W=\frac{E}{I}$ tend vers une limite $W_{0} ;$ si, de plus, on admet qu'aux vitesses suffisamment élevées, l'énergie moyenne d'ionisation ne dépend pas de la nature de la particule incidente, mais uniquement de sa vitesse, il apparaît que l'énergie moyenne d'ionisation We déterminée pour des électrons de quelques $\mathrm{keV}$ doit approcher beaucoup plus la limite $W_{0}$ que l'énergie moyenne d'ionisation $W \alpha$ déterminée pour les particules alpha. En posant $W_{0}=W e$, il vient $\Delta=\frac{W_{\alpha}-W_{\mathrm{e}}}{W_{\alpha}} E_{\alpha}$.

En utilisant les mesures de Jesse et Sadauskis (Phys. Res., 1955, 97, 1668) on a :

$$
\begin{array}{lccl}
\text { Argon } & W_{\alpha}=W_{\mathrm{e}} & \text { par convention } & \Delta=0 \\
\text { Air } & W_{\alpha}=35,6 & W_{\mathrm{e}}=34,1 & \Delta=210 \mathrm{keV} \\
\text { Azote } & W_{\alpha}=36,6 & W_{\mathrm{e}}=34,7 & \Delta=280 \mathrm{keV}
\end{array}
$$

